\title{
In Vivo Expression of Cyclooxygenase-1 in Activated Microglia and Macrophages During Neuroinflammation Visualized by PET with ${ }^{11} \mathrm{C}$-Ketoprofen Methyl Ester
}

\author{
Miho Shukuri ${ }^{1,2}$, Misato Takashima-Hirano ${ }^{1}$, Keiko Tokuda $^{1}$, Tadayuki Takashima ${ }^{1}$, Kiyoshi Matsumura ${ }^{3}$, Osamu Inoue ${ }^{2}$, \\ Hisashi Doi ${ }^{1}$, Masaaki Suzuki ${ }^{1}$, Yasuyoshi Watanabe ${ }^{1}$, and Hirotaka Onoe ${ }^{1}$ \\ ${ }^{I}$ RIKEN Center for Molecular Imaging Science, Hyogo, Japan; ${ }^{2}$ Division of Health Sciences, Graduate School of Medicine, Osaka \\ University, Osaka, Japan; and ${ }^{3}$ Department of Biomedical Engineering, Osaka Institute of Technology, Osaka, Japan
}

Cyclooxygenase (COX)-1 and -2 are prostanoid-synthesizing enzymes that play important roles in the regulation of neuroinflammation and in the development of neurodegenerative disorders. However, the specific functions of these isoforms are still unclear. We recently developed ${ }^{11} \mathrm{C}$-labeled ketoprofen methyl ester as a PET probe that targets the COXs for imaging neuroinflammation, though its responsible isoform is yet to be determined. In the present study, we performed ex vivo and in vivo imaging studies with ${ }^{11} \mathrm{C}$-ketoprofen methyl ester and determined the contributions of the COX isoforms during the neuroinflammatory process. Methods: To identify the COX isoform responsible for ${ }^{11} \mathrm{C}$-ketoprofen methyl ester in the brain, we examined the ex vivo autoradiography of ${ }^{11} \mathrm{C}$-ketoprofen methyl ester using COX-deficient mice. Time-dependent changes in accumulation of ${ }^{11} \mathrm{C}$-ketoprofen methyl ester during the neuroinflammatory process were evaluated by PET in rats with hemispheric neuroinflammation induced by intrastriatal injection of lipopolysaccharide or quinolinic acid. In both rat models, cell-type specificity of COX isoform expression during neuroinflammation was identified immunohistochemically. Results: Ex vivo autoradiographic analysis of COX-deficient mice revealed a significant reduction of ${ }^{11} \mathrm{C}$-ketoprofen methyl ester accumulation only in COX-1-deficient mice, not COX-2-deficient mice. PET of rats after intrastriatal injection of lipopolysaccharide showed a significant increase in accumulation of ${ }^{11} \mathrm{C}$-ketoprofen methyl ester in the inflamed area. This increase was evident at the early phase of $6 \mathrm{~h}$, peaked at day 1 , and then returned to basal levels by day 7 . In addition, immunohistochemical analysis revealed that the population of activated microglia and macrophages was elevated at the early phase with COX-1 expression but not COX-2. A significant increase in ${ }^{11} \mathrm{C}$-ketoprofen methyl ester accumulation was also observed at day 1 after intrastriatal injection of quinolinic acid, with increased COX-1-expressing activated microglia and macrophages. Conclusion: We have identified ${ }^{11} \mathrm{C}$-ketoprofen methyl ester as a COX-1-selective PET probe, and using this, we have also demonstrated a time-dependent expression of COX-1 in activated microglia and macrophages during the neuroinflammatory process in the living brain. Thus, COX-1 may play a crucial role in

Received Oct. 13, 2010; revision accepted Feb. 28, 2011.

For correspondence or reprints contact: Hirotaka Onoe, RIKEN Center for Molecular Imaging Science, 6-7-3 Minatojima Minamimachi, Chuo-ku, Kobe, Hyogo 650-0047, Japan.

E-mail: hiro.onoe@riken.jp

COPYRIGHT @ 2011 by the Society of Nuclear Medicine, Inc. the pathology of neuroinflammation and might be a critical target for the diagnosis and therapy of neurodegenerative disorders.

Key Words: molecular imaging; PET; NSAIDs; arachidonic acid cascade; innate immunity

J Nucl Med 2011; 52:1094-1101

DOI: 10.2967/jnumed.110.084046

$\mathbf{N}$ euroinflammation is an important mechanism in defense responses to pathogenic events but is also recognized as a key component in the progression of neurodegenerative diseases such as Alzheimer disease, Parkinson disease, amyotrophic lateral sclerosis, multiple sclerosis, traumatic brain injury, and HIV encephalitis $(1,2)$. Cyclooxygenase $(\mathrm{COX})$ is a key enzyme in the rate-limiting conversion of arachidonic acid to prostaglandins, one of the proinflammatory factors, and is known to play a crucial role in the inflammatory cascade $(3,4)$. Two distinct COX isoforms, COX-1 and COX-2, have been characterized. COX-1 is classically considered a constitutively expressed housekeeping enzyme and COX-2 an inflammatory inducible enzyme $(5,6)$. In the central nervous system, overexpression of COX-1 and COX-2 in patients with ischemic, traumatic brain injury and in several neurodegenerative diseases such as Alzheimer disease, Parkinson disease, and amyotrophic lateral sclerosis has been reported, indicating that COXmediated neuroinflammation is a critical component in neuronal degeneration (7-11).

A noninvasive in vivo imaging method to monitor COX expression by PET could be a valuable tool for investigating the role of the COX isoforms in the pathologic processes of neurologic disorders (12). COX inhibitors such as nonsteroidal antiinflammatory drugs labeled with ${ }^{18} \mathrm{~F}$ or ${ }^{11} \mathrm{C}$ have been evaluated as PET probes for COX imaging in inflammation. Previously, PET probes that are highly selective to COX-2, such as ${ }^{18} \mathrm{~F}$-desbromo-Dup697 and ${ }^{11} \mathrm{C}$-celecoxib, had been developed $(13,14)$. However, the selectivity of these probes to $\mathrm{COX}-2$ proved to be 
unsatisfactory in vivo when examined by PET. Recently, we developed the use of ${ }^{11} \mathrm{C}$-ketoprofen methyl ester (the acidic form specific to COX-1; 50\% inhibition concentrations, $0.047 \mu \mathrm{M}$ for COX-1 and $2.9 \mu \mathrm{M}$ for COX-2) together with a method for efficient, rapid ${ }^{11} \mathrm{C}$-labeling of 2-arylpropionic acids and their esters (a well-known class of nonsteroidal antiinflammatory drugs) as a PET probe for COX imaging of neuroinflammation (15). A significant and specific accumulation in the inflamed region of rat brain caused by intracranial lipopolysaccharide injection was shown by ${ }^{11} \mathrm{C}$-ketoprofen methyl ester but not ${ }^{11} \mathrm{C}$-ketoprofen because of blood-brain barrier permeability (15).

In the present study, we performed PET with ${ }^{11} \mathrm{C}$-ketoprofen methyl ester to examine time-dependent changes in the contribution of the COX isoforms during the neuroinflammatory processes of 2 different types of pathologic neuronal damage induced by intrastriatal injection of lipopolysaccharide and quinolinic acid. Lipopolysaccharide is a cell-wall component of gram-negative bacteria that is widely used for activating the innate immune system and is reported to cause neuroinflammation with microglial activation $(16,17)$. Quinolinic acid is an excitotoxic $N$ methyl-D-aspartate receptor agonist reported to induce the death of neurons and astrocytes with excessive induction of nitric oxide and nitric oxide-mediated free radicals $(18,19)$. In this study, we also characterized the selectivity and specificity of ${ }^{11} \mathrm{C}$-ketoprofen methyl ester to COX-1 and COX-2 in vivo using $\mathrm{COX}-1-$ deficient $\left(\mathrm{COX}^{-1} 1^{-1}\right)$ and COX-2-deficient $\left(\mathrm{COX}-2^{-I-}\right)$ mice, respectively, and identified immunohistochemically the cell-type specificity of COX isoform expression.

\section{MATERIALS AND METHODS}

\section{Radiochemistry}

${ }^{11} \mathrm{C}$-labeled ketoprofen methyl ester was synthesized using a rapid ${ }^{11} \mathrm{C}$-methylation method recently developed by our group (15). The specific radioactivity ranged from 19 to $47 \mathrm{GBq} / \mu \mathrm{mol}$ at the end of synthesis. Both the radiochemical and the chemical purities were greater than $99 \%$ as determined by high-performance liquid chromatography (COSMOSIL 5C 18 -AR-II column [Nacalai Tesque, Inc.]; 4.6-mm internal diameter $\times 100 \mathrm{~mm}$; particle size, $5 \mu \mathrm{m}$; mobile phase, 50:50 acetonitrile:water; flow rate, $1 \mathrm{~mL} / \mathrm{min}$; ultraviolet detection, $254 \mathrm{~nm}$; retention time, $5 \mathrm{~min})$.

${ }^{11} \mathrm{C}$-labeled ( $R$ )- $N$-methyl- $N$-(1-methylpropyl)-1-(2-chlorophenyl) isoquinoline-3-carboxamide [ $R$-PK11195] was synthesized according to the method described by Shah et al. (20), with slight modifications. The specific activity ranged from 44 to $135 \mathrm{GBq} / \mu \mathrm{mol}$ at the end of synthesis. Both the radiochemical and the chemical purities were greater than $99 \%$ as determined by high-performance liquid chromatography (COSMOSIL $5 \mathrm{C}_{18}$-AR-II column; 4.6-mm internal diameter $\times 100 \mathrm{~mm}$; particle size, $5 \mu \mathrm{m}$; mobile phase, 65:35 acetonitrile:water; flow rate, $1 \mathrm{~mL} / \mathrm{min}$; ultraviolet detection, $215 \mathrm{~nm}$; retention time, $4 \mathrm{~min}$ ).

\section{Animals}

All experimental protocols were approved by the Animal Care and Use Committee of RIKEN Kobe Institute (MAH19-03) and were performed in accordance with the National Institutes of
Health principles of laboratory animal care (21). Male mice homozygous-deficient $(-/-)$ for COX-1 and COX-2 (3-4 mo of age) bred on a C57BL/6 $\times 129 /$ Ola (C57/129) background were obtained from Taconic Farms Inc. These mice and their matching wild-type littermate controls were produced by crossing COX-1 or COX-2 heterozygous mice that had been maintained for more than 35 generations (22,23). Male Sprague-Dawley rats (3 mo old) were obtained from CLEA Japan, Inc. Four to 5 mice were housed in a cage, under a 12-h light-dark cycle (lights off at 20:00) at $23^{\circ} \mathrm{C} \pm 1{ }^{\circ} \mathrm{C}$ and $60 \% \pm 5 \%$ humidity and were allowed access to food and water ad libitum.

\section{Generation of Neuroinflammatory Models}

Injection of lipopolysaccharide or quinolinic acid into the striatum of rats was performed using a stereotactic apparatus (SR-6N; Narishige Co.) under sodium pentobarbital anesthesia $(50 \mathrm{mg} / \mathrm{kg})$. Lipopolysaccharide from Escherichia coli 026:B6 (0.5 $\mu \mathrm{g} / \mu \mathrm{L}$; Sigma-Aldrich Co.) or quinolinic acid $(60 \mathrm{nmol} / \mu \mathrm{L}$; SigmaAldrich Co.) was dissolved in saline and injected into the striatum using a $10-\mu \mathrm{L}$ syringe with a 26 -gauge needle controlled by an automated syringe pump (Muromachi Kikai Co.) at a rate of $0.2 \mu \mathrm{L} / \mathrm{min}$ for $5 \mathrm{~min}$. The coordinates for the stereotactic injection were $+0.2 \mathrm{~mm}$ anterior/posterior, $+3.2 \mathrm{~mm}$ lateral, and $-5.5 \mathrm{~mm}$ dorsal/ventral from the bregma, according to the atlas of Paxinos and Watson (24). After the injection, the needle was kept in the same position for an additional $5 \mathrm{~min}$ and then retrieved slowly from the brain.

\section{PET Studies}

A venous catheter for PET probe injection was inserted into the tail vein before PET. The rats were anesthetized with a mixture of $1.5 \%$ isoflurane and 7:3 nitrous oxide:oxygen and placed on the bed of a small-animal PET scanner (microPET Focus 220; Siemens). The PET scanner has a spatial resolution of $1.4 \mathrm{~mm}$ in full width at half maximum at the center of the field of view (220 mm in diameter) and an axial extent at $78 \mathrm{~mm}$. ${ }^{11}$ C-ketoprofen methyl ester or ${ }^{11} \mathrm{C}-\mathrm{PK} 11195$ ( $\sim 70 \mathrm{MBq}$ per animal) dissolved in $1 \mathrm{~mL}$ of saline was injected via the cannula inserted into the tail vein for $10 \mathrm{~s}$, and emission data were then acquired for 45 min using a 3D list-mode method. The data were reconstructed with standard 2-dimensional filtered backprojection (ramp filter, cutoff frequency at 0.5 cycles per pixel). Regions of interest were placed on the ipsilateral and contralateral sides of the striatum using image processing software (PMOD, version 3.0; PMOD Technologies Ltd.) according to the fused PET/MR T1-weighted image (Supplemental Fig. 1; supplemental materials are available online only at http://jnm. snmjournals.org). Regional uptake of radioactivity in the brain was decay-corrected to the injection time and was expressed as the standardized uptake value (SUV), where SUV = tissue radioactivity concentration $\left(\mathrm{MBq} / \mathrm{cm}^{3}\right) /$ injected radioactivity $(\mathrm{MBq}) \times$ body weight $(\mathrm{g})$.

\section{Ex Vivo Autoradiography}

To assess the quantification of emission data, we performed ex vivo brain slice autoradiography. Because a rapid and transient brain time-activity curve was observed in our preliminary ${ }^{11} \mathrm{C}$ ketoprofen methyl ester PET study with mice, we decided to use the time point at $15 \mathrm{~min}$ to avoid the effect of cerebral blood flow. Fifteen minutes after injection of ${ }^{11} \mathrm{C}$-ketoprofen methyl ester ( $\sim 20 \mathrm{MBq}$ per animal), the mice were sacrificed under deep anesthesia with $3.0 \%$ isoflurane and the brain was sliced into coronal 
sections ( $1 \mathrm{~mm}$ thick) using a brain matrix (RBM-2000C; ASI instruments, Inc.) on ice. The coronal slices were placed on an imaging plate (BAS-SR2040; Fuji Photo Film Co.) for $1 \mathrm{~h}$. The exposed imaging plate was then scanned with a bioimaging analyzer (FLA-7000IR; Fuji Photo Film Co.). To obtain quantitative data from ex vivo autoradiograms, we manually placed regions of interest on the brain regions according to the digital photograph obtained from a photo scanner. Brain regional radioactivity (photostimulable luminescence $/ \mathrm{mm}^{2}$ ) was calibrated to the uptake value $(\%$ dose $/ g)$, which was defined as $100 \times$ tissue activity concentration $(\mathrm{MBq} / \mathrm{g}) /$ injected dose $(\mathrm{MBq})$ using whole-brain radioactivity measured by a $\gamma$-well counter (Wizard 1480; PerkinElmer, Inc.).

\section{Histology}

The mice were deeply anesthetized and perfused transcardially with ice-cold $0.9 \%$ saline followed by $4 \%$ paraformaldehyde solution. Their brains were removed and afterward were fixed with formalin solution for $6 \mathrm{~h}$, placed in a $30 \%$ sucrose solution, and embedded in optimal-cutting-temperature compound (Sakura Finetek Japan Co.). Serial coronal sections (25 $\mu \mathrm{m}$ thick) were obtained with a cryostat and thaw-mounted on glass slides. The rats were perfused with ice-cold $0.9 \%$ saline under deep anesthesia and their brains were rapidly frozen in dry ice powder. The brain sections (20 $\mu \mathrm{m}$ thick) were obtained with a cryostat and thawmounted on glass slides. For immunostaining, the sections were treated with $2 \%$ paraformaldehyde in $0.1 \mathrm{M}$ phosphate-buffered saline for $10 \mathrm{~min}$.

The antibodies used in this study were rabbit IgG (1:500; Cayman Chemical Co.) for COX-1, rabbit IgG (1:500; Cayman Chemical Co.) for COX-2, and rabbit $\operatorname{IgG}(1: 1,000$; developed in our laboratory) for the $18-\mathrm{kDa}$ translocator protein.

To characterize cell type, we double-stained the COX-1-positive, COX-2-positive, and translocator protein-positive cells with either the activated microglia/macrophage marker (1:1,000 CD11b, clone OX-42, mouse IgG; AbD Serotec), the astrocyte marker (1:1,000 glial fibrillary acidic protein [GFAP], mouse $\mathrm{IgG}$; Chemicon International, Inc.), or the neuronal marker (1:1,000 neuron-specific nuclear protein, mouse IgG; Chemicon International, Inc.). After overnight incubation of the brain sections with the mixed antibodies at room temperature, the primary antibodies were visualized with either a Cy3-labeled secondary antibody (1:500; Jackson ImmunoResearch) or Alexa Fluor 488 (1:500; Invitrogen). Fluorescent images were captured with a 3laser confocal microscope (Olympus Optical Co.). The number of immunoreactive cells per section was quantified by counting the number of COX-1-, COX-2-, and OX-42-stained cells and COX$1-/ \mathrm{OX}-42-$ costained cell bodies within a $0.4-\mathrm{mm}^{2}$ area of the striatum.

\section{Statistical Analysis}

Statistical analysis was performed using the Student $t$ test. $P$ values less than 0.05 were considered to be statistically significant.

\section{RESULTS}

\section{Accumulation of ${ }^{11} \mathrm{C}-K e t o p r o f e n$ Methyl Ester in Brain of $\mathrm{COX}-1^{-1-}$ and $\mathrm{COX}-2^{-1-}$ Mice}

To determine the selectivity of ${ }^{11} \mathrm{C}$-ketoprofen methyl ester to the $\mathrm{COX}$ isoforms, we performed ${ }^{11} \mathrm{C}$-ketoprofen methyl ester brain imaging on COX-1- and COX-2-deficient mice by ex vivo autoradiography. High accumulations of ${ }^{11} \mathrm{C}$-ketoprofen methyl ester in the hippocampus, cerebral cortex, and cerebellum were observed in wild-type $\left(\mathrm{COX}-1^{+/+}, \mathrm{COX}-2^{+/+}\right)$mice, consistent with previous immunohistochemical studies on COX-1 and COX-2 expression (Fig. 1). In all brain regions, accumulation of ${ }^{11} \mathrm{C}$-ketoprofen methyl ester was significantly lower in $\mathrm{COX}-1^{-/-}$mice than in wild-type $\left(\mathrm{COX}-1^{+/+}\right)$mice (Fig. 1A). In contrast, no difference in accumulation was observed between $\mathrm{COX}-2^{-1-}$ and wild-type $\left(\mathrm{COX}-2^{+/+}\right)$ mice (Fig. 1B), indicating that ${ }^{11} \mathrm{C}$-ketoprofen methyl ester selectively recognizes COX-1 in the brain. Lack of COX-1 and COX-2 proteins in the brains of $\mathrm{COX}-1^{-1-}$ and COX$2^{-1-}$ mice, respectively, was confirmed by immunohistochemistry (Supplemental Fig. 2).

\section{Time-Dependent Changes of ${ }^{11} \mathrm{C}$-Ketoprofen Methyl Ester Accumulation in Lipopolysaccharide-Induced Neuroinflammation}

Time-dependent changes of ${ }^{11} \mathrm{C}$-ketoprofen methyl ester after intrastriatal injection of lipopolysaccharide in rats were investigated and were compared with those of ${ }^{11} \mathrm{C}$ PK11195, a PET probe for activated glial cells. After the lipopolysaccharide injection, a significant increase of ${ }^{11} \mathrm{C}$ ketoprofen methyl ester accumulation in the ipsilateral striatum was observed at $6 \mathrm{~h}$ and at day 1 , which is when the accumulation reached its highest level $(\sim 4$-fold higher than
FIGURE 1. Accumulation of ${ }^{11} \mathrm{C}$-ketoprofen methyl ester in brain of $\mathrm{COX}-1^{-1-}$ and $\mathrm{COX}-2^{-1-}$ mice. Ex vivo autoradiography and regional brain tissue uptake of ${ }^{11} \mathrm{C}$ ketoprofen methyl ester in $\mathrm{COX}-1^{+/+}$and $\mathrm{COX}-1^{-1-}$ mice (A) and $\mathrm{COX}-2^{+/+}$and $\mathrm{COX}-2^{-1-}$ mice (B) was obtained $15 \mathrm{~min}$ after injection of ${ }^{11} \mathrm{C}$-ketoprofen methyl ester. F-CTX = frontal cortex; P-CTX = parietal cortex; STR = striatum; HIP = hippocampus; CERE $=$ cerebellum. Data are mean $\pm \mathrm{SD}(n=6)$. ${ }^{\star \star \star} P<0.001$ vs. wildtype mice.
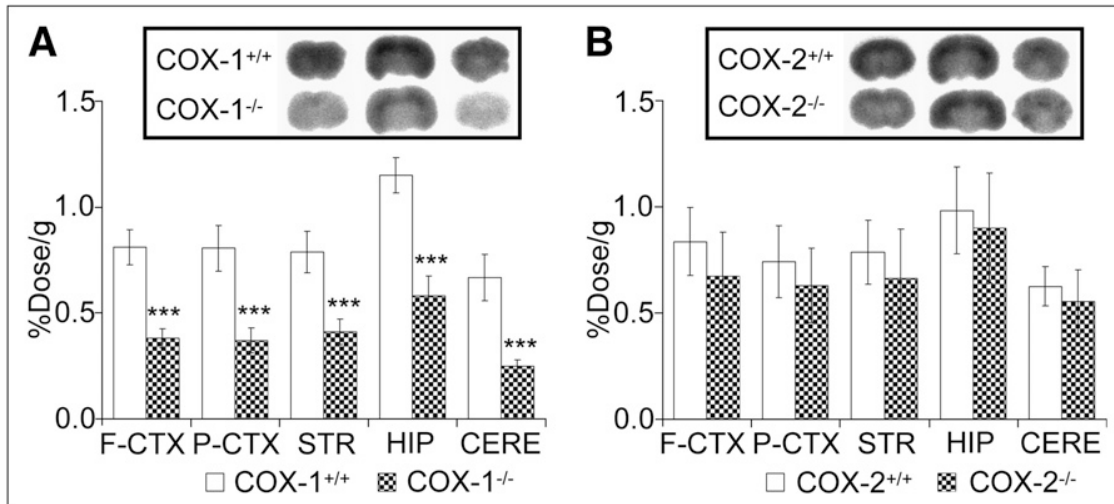
in the contralateral striatum) (Figs. 2A and 2B). At $7 \mathrm{~d}$ after the lipopolysaccharide injection, the accumulation of ${ }^{11} \mathrm{C}$ ketoprofen methyl ester returned to the basal preinjection level. A slight but significant increase in ${ }^{11} \mathrm{C}$-ketoprofen methyl ester accumulation was observed only at $6 \mathrm{~h}$ in the site of the saline injection (Supplemental Fig. 3), suggesting that needling may drive increased permeability of the blood vessel as an acute inflammatory reaction. On the other hand, a significant accumulation of ${ }^{11}$ C-PK11195 in the ipsilateral striatum was observed first at day 1 , followed by the highest accumulation at day 3 ( $\sim 2$-fold), and this level lasted until at least day 14 (Figs. 2A and 2C).

\section{Identification of Cell Type Associated with COX Expression in Lipopolysaccharide-Induced Neuroinflammation}

To identify the cell type associated with ${ }^{11} \mathrm{C}$-ketoprofen methyl ester accumulation in lipopolysaccharide-induced neuroinflammation, we examined double immunostaining of COX-1 and COX-2 with the expression of OX-42, GFAP, and neuron-specific nuclear protein in activated microglia/ macrophages, astrocytes, and neurons, respectively. Activation of microglia and macrophages was seen at $6 \mathrm{~h}$ and at day 1 after the lipopolysaccharide injection (Fig. 3A). In contrast, though activation of astrocytes was also seen at $6 \mathrm{~h}$ after the lipopolysaccharide injection, it lasted to at least 14 $\mathrm{d}$, with a gradual increase in staining intensity. Expression of COX-1 was found in the neurons and activated microglia and macrophages but not in astrocytes (Fig. 3B and Supplemental Fig. 4A). On the other hand, COX-2 expression was observed only in neurons, not in microglia, macro- phages, or astrocytes, even after the lipopolysaccharide injection (Fig. 3C and Supplemental Fig. 4B). However, no significant neuronal damage was observed in the core region of the lipopolysaccharide injection site. The population of COX-1-expressing cells was significantly increased, and this increase corresponded to the increased level of activated microglia and macrophages (Fig. 4A). Alternatively, the population of COX-2-expressing cells did not show any changes at any time points examined. The time course of appearance of COX-1-expressing activated microglia and macrophages after the lipopolysaccharide injection closely corresponded to that of ${ }^{11} \mathrm{C}$-ketoprofen methyl ester accumulation in the injection site of the brain (Fig. 4B), whereas Western blot analysis showed little increase in total protein level of $70-\mathrm{kDa}$ COX-1 in the lipopolysaccharide-injected hemisphere (1.02 \pm 0.12 -fold from the contralateral side, $n=3$ ) (Supplemental Fig. 5).

\section{Accumulation of ${ }^{11} \mathrm{C}$-Ketoprofen Methyl Ester in Quinolinic Acid-Induced Excitotoxic Neurodegeneration}

To investigate the possible involvement of COX-1 in the process of excitotoxic neurodegeneration, we performed PET studies with ${ }^{11} \mathrm{C}$-ketoprofen methyl ester using intrastriatal quinolinic acid-injected rats. Similar to the results of lipopolysaccharide, injecting quinolinic acid also induced a high accumulation of ${ }^{11} \mathrm{C}$-ketoprofen methyl ester in the ipsilateral striatum at day 1 after injection (Fig. 5). Though the level of ${ }^{11} \mathrm{C}$-ketoprofen methyl ester accumulation induced by quinolinic acid (SUV, $0.75 \pm 0.09$ ) was lower than that observed with lipopolysaccharide (SUV,

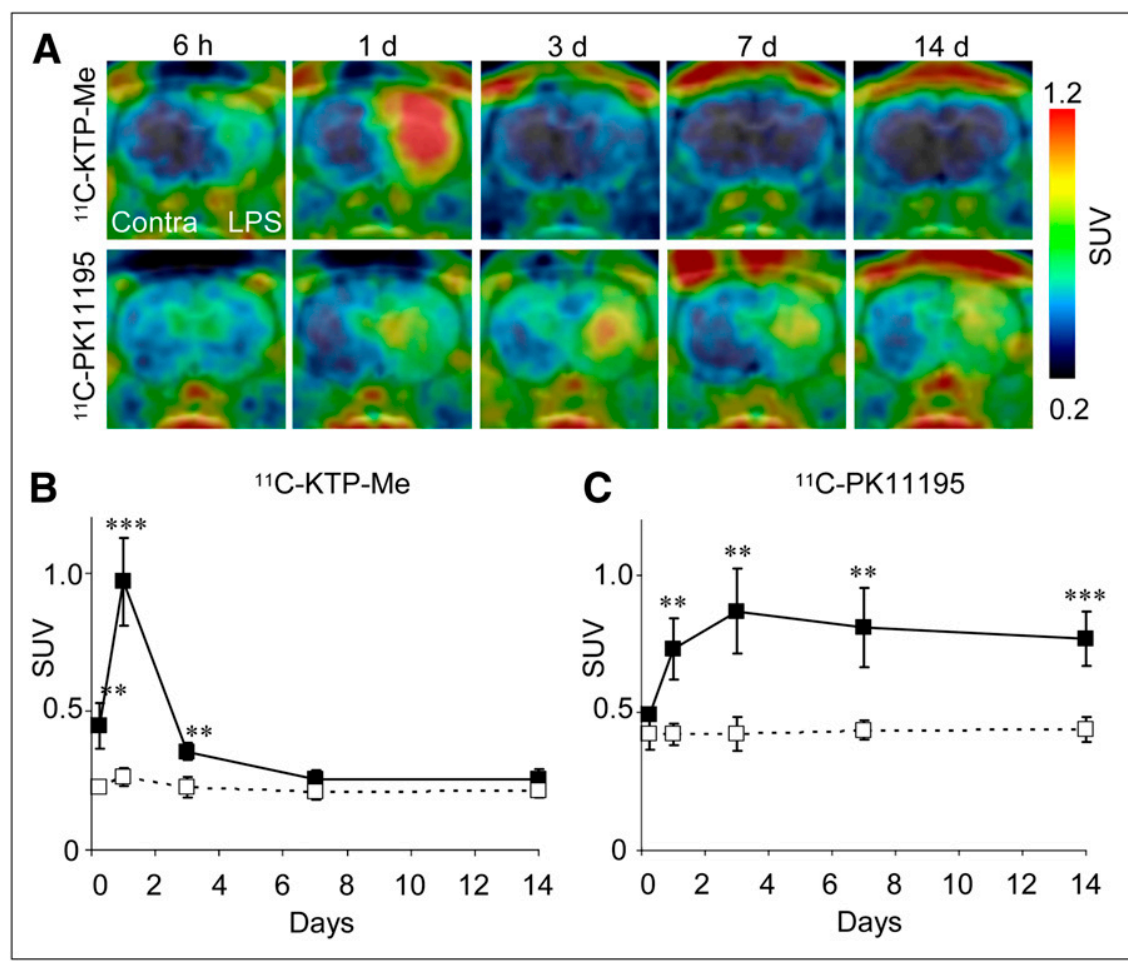

FIGURE 2. Time-radioactivity changes in ${ }^{11} \mathrm{C}$-ketoprofen methyl ester and ${ }^{11} \mathrm{C}$ PK11195 accumulation during lipopolysaccharide-induced neuroinflammation. (A) Summed PET image of ${ }^{11} \mathrm{C}$-ketoprofen methyl ester and ${ }^{11} \mathrm{C}-\mathrm{PK} 11195$ from 5 to $45 \mathrm{~min}$ in rat brain with hemispheric neuroinflammation induced by intrastriatal lipopolysaccharide $(0.5-\mu \mathrm{g})$ injection. "Contra" and "LPS" mean contralateral and lipopolysaccharide-injected sides, respectively. (B) Quantification of radioactivity of ${ }^{11} \mathrm{C}$-ketoprofen methyl ester in contralateral striatum $(\square)$ and lipopolysaccharide-injected striatum ( $\square$ ). (C) Quantification of radioactivity of ${ }^{11} \mathrm{C}-\mathrm{PK} 11195$ in contralateral striatum $(\square)$ and lipopolysaccharide-injected striatum ( $\square$ ). Data are SUV mean \pm SD $(n=4$, 2 of which are obtained from longitudinal PET studies throughout experimental period). $\mathrm{KTP}-\mathrm{Me}=$ ketoprofen methyl ester. ${ }^{\star \star} P<$ 0.01 vs. contralateral striatum. ${ }^{\star \star \star} P<0.001$ vs. contralateral striatum. 
FIGURE 3. Time courses of activation of glial cells and cell-specific expression of COX-1 and COX-2 in lipopolysaccharideinduced neuroinflammation. (A) Representative photomicrographs of OX-42 and GFAP immunohistochemistry in striatum after lipopolysaccharide $(0.5-\mu \mathrm{g})$ injection. (B) Representative photomicrographs of double immunofluorescent labeling of COX-1 (green) and neuron-specific nuclear protein (a and $\mathrm{d}$; red) and OX-42 (b and e; red) or GFAP (c and $\mathrm{f}$; red) in striatum at day 1 after lipopolysaccharide $(0.5-\mu \mathrm{g})$ injection. In contralateral striatum $(\mathrm{a}-\mathrm{c})$, only neuronal cells with expression of COX-1 are present (arrows in a). In lipopolysaccharide-injected striatum (d-f), COX-1 is expressed in neuronal cells (arrows in d) and in activated microglia and macrophages (arrows in e) but not in astrocytes (f). (C) Representative photomicrographs of double immunofluorescent labeling of COX-2 (green) and neuron-specific nuclear protein ( $\mathrm{a}$ and $\mathrm{d}$; red), and $\mathrm{OX}-42$ (b and e; red) or GFAP (c and f; red) in striatum at

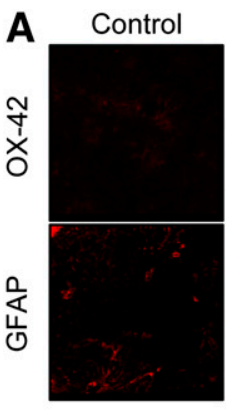

$6 \mathrm{~h}$

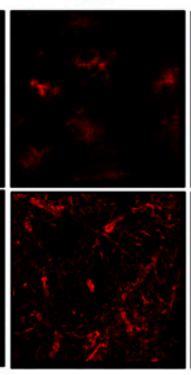

B
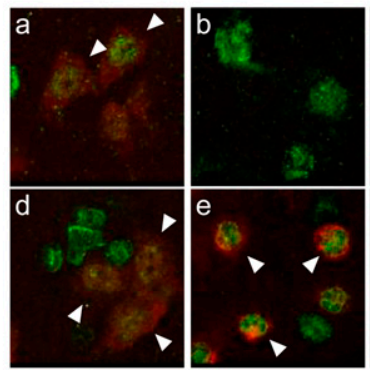

$1 d$
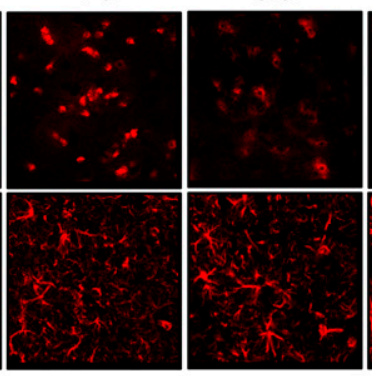

C

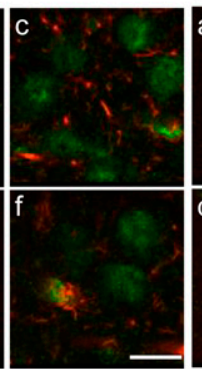

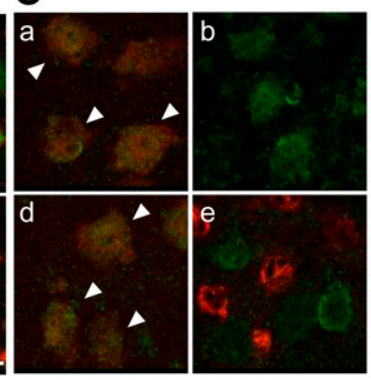

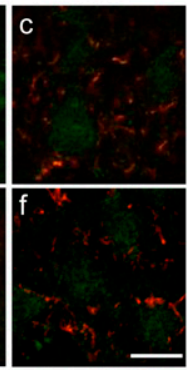

day 1 after lipopolysaccharide $(0.5-\mu \mathrm{g})$

injection. In contralateral striatum (a-c), only neuronal cells with expression of COX-2 are present (arrows in a). In lipopolysaccharideinjected striatum (d-f), COX-2 is also expressed in neuronal cells (arrows in d) but not in activated microglia and macrophages (e) or astrocytes (f). Scale bars $=50 \mu \mathrm{m}$ (in A) and $10 \mu \mathrm{m}$ (in B and C).

$0.97 \pm 0.16$ ), the ratio of ${ }^{11} \mathrm{C}$-ketoprofen methyl ester to the contralateral striatum reached approximately 4 -fold.

\section{COX Expression in Quinolinic Acid-Induced Neurodegeneration}

To identify the expression of COX-1 and COX-2 and their cell type specificity in quinolinic acid-induced excitotoxic neurodegeneration, we performed immunohistochemical analysis of brain samples at day 1 after injection, at which time the number of COX-1-expressed microglia and macrophages peaked to the same level as that of lipopolysaccharide. Quinolinic acid injection produced substantial amounts of neuronal cell loss in the core of the injection site and decreased to approximately $52 \%$ in neuron-specific nuclear protein-positive cells, with activation of astrocytes, and microglia and macrophages detected around the injection site. Most of the activated microglia and macrophages showed COX-1 expression, yet the astrocytes showed no COX-1 or COX-2 expression (Supplemental Fig. 6).

\section{DISCUSSION}

In this study, we demonstrated that ${ }^{11} \mathrm{C}$-ketoprofen methyl ester is a suitable PET probe for detecting and monitoring COX-1 expression or activity during neuroinflammation. In addition, by performing PET studies with ${ }^{11} \mathrm{C}$-ketoprofen methyl ester, we identified the contribution of COX-1 in activated microglia and macrophages during neuroinflammation in vivo.

Previously, PET probes selective for COX-2 rather than COX-1 have been the focus of imaging the inflammatory process, because COX-2 is known to be induced by a variety of inflammatory stimuli, including endotoxins, cytokines, and growth factors (12). However, there has been no successful imaging of inflammation models $(13,14)$. In this
FIGURE 4. Population of immunoreactive cells in striatum after lipopolysaccharide injection. (A) Cells that express COX-1, COX-2, and OX-42 in striatum at day 1 after lipopolysaccharide $(0.5-\mu \mathrm{g})$ injection. (B) Time-dependent changes in COX-1/OX42-positive cells in striatum after lipopolysaccharide injection. Numbers of cells in contralateral striatum ( $\square$ ) and in lipopolysaccharide-injected striatum $(\square)$ are shown. Data are expressed as cell numbers (counts $\left./ 0.4 \mathrm{~mm}^{2}\right)$ and mean $\pm \mathrm{SD}(n=4)$. ${ }^{\star \star \star} P<0.001$ vs. contralateral striatum.
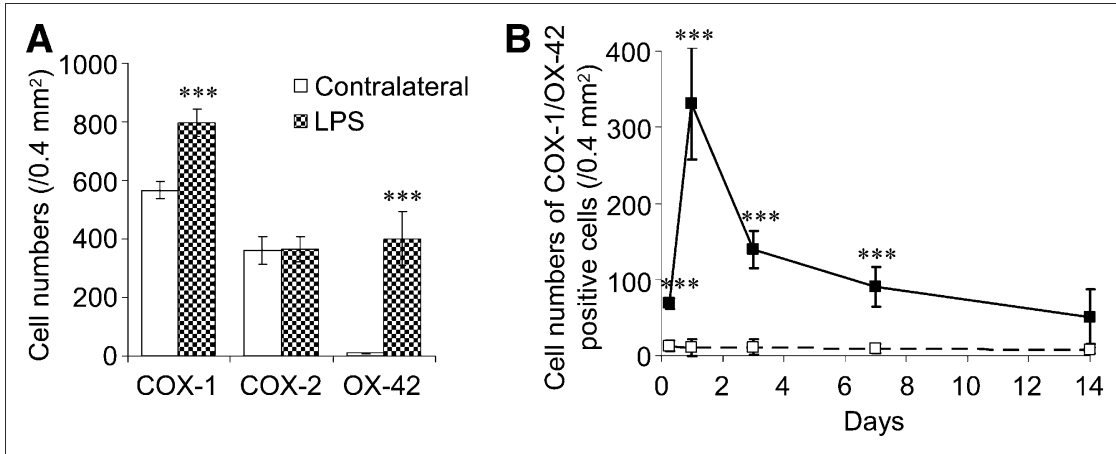


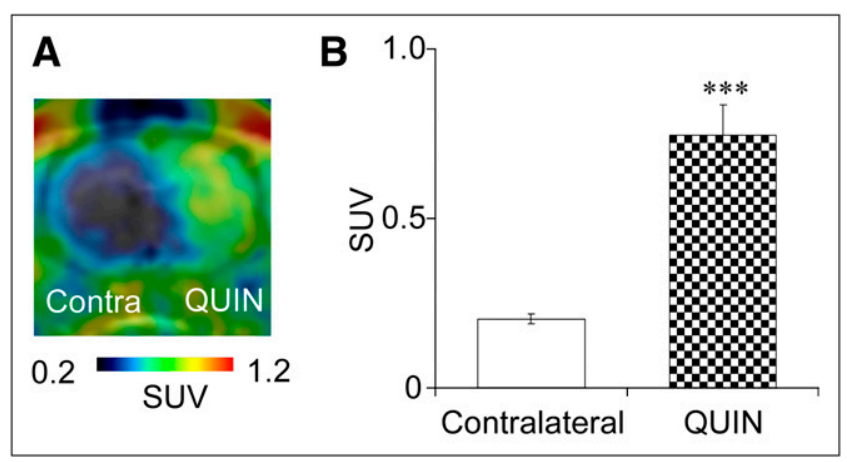

FIGURE 5. Changes in ${ }^{11} \mathrm{C}$-ketoprofen methyl ester accumulation in excitotoxic neurodegeneration induced by quinolinic acid. (A) Summed PET image from 5 to 45 min after ${ }^{11} \mathrm{C}$-ketoprofen methyl ester administration in rat brain $1 \mathrm{~d}$ after quinolinic acid (60-nmol) injection. "Contra" and "QUIN" mean contralateral and quinolinic acid-injected sides, respectively. (B) Quantification of radioactivity of ${ }^{11} \mathrm{C}$-ketoprofen methyl ester in contralateral and quinolinic acidinjected striatum. Data are expressed as SUV and mean \pm SD $(n=$ 3). ${ }^{\star \star *} P<0.001$ vs. contralateral striatum.

study, lipopolysaccharide and quinolinic acid were shown to induce a significantly high accumulation of ${ }^{11} \mathrm{C}$-ketoprofen methyl ester, which we developed recently, in the injection site of the rat brain. To the best of our knowledge, ${ }^{11} \mathrm{C}$-ketoprofen methyl ester is the first PET probe targeting COX-1 in neuroinflammation under in vivo conditions.

Ketoprofen, produced by hydrolysis of ketoprofen methyl ester, has a higher affinity to COX-1 than to COX-2, whereby $50 \%$ inhibition concentrations in human whole blood cells are 0.047 and $2.9 \mu \mathrm{M}$, respectively (25). However, the selectivity of ${ }^{11} \mathrm{C}$-ketoprofen methyl ester to each $\mathrm{COX}$ isoform under in vivo imaging conditions has not yet been clearly determined. The present ex vivo autoradiographic study demonstrated that the accumulation of ${ }^{11} \mathrm{C}$-ketoprofen methyl ester is significantly low in COX$1^{-1-}$ mice yet in $\mathrm{COX}-2^{-1-}$ does not differ from that in wild-type mice. Therefore, most of the accumulation of ${ }^{11} \mathrm{C}$-ketoprofen methyl ester in the brain should be derived from its binding to COX-1. In addition to these COX isoforms, a new splice variant of COX-1, referred to as COX3 , which is selectively inhibited by acetaminophen and antipyrine, has been reported (26). Though the selectivity of ${ }^{11} \mathrm{C}$-ketoprofen methyl ester to COX-3 is not clear, the effect of ketoprofen on methamphetamine-induced hyperthermia in mice has been reported to be different from that of antipyrine (27).

The activation of microglia and macrophages was observed only at the early phase of neuroinflammation, with the largest population observed at day 1 after intrastriatal injection of lipopolysaccharide. Our double immunostaining studies revealed that most of the activated microglia and macrophages $(82 \%)$ expressed COX-1 but not COX-2. In contrast, astrocytes did not show any expression of either COX-1 or COX-2 throughout the entire neuroinflammation. Though COX-1 is ubiquitously expressed in neuronal cells under normal conditions, any changes in the neuronal cell population and localization of COX-1 in these cells were evident in lipopolysaccharide-induced neuroinflammation. The increased number of COX-1-positive cells in the lipopolysaccharide-injected striatum closely matched that of activated microglia and macrophages. Therefore, we consider that COX-1 may be newly induced with microglial activation or that COX-1-positive macrophages may be infiltrated to the parenchyma during the neuroinflammatory process. Indeed, both proliferation of microglia and invasion of macrophages have been demonstrated in the brain during neuroinflammation induced by the intraperitoneal or intrastriatal injection of lipopolysaccharide in mice $(28,29)$.

We identified the expression of COX-2 only in neuronal cells, and induction of COX-2 expression was not observed in any brain cells at any time points after lipopolysaccharide injection, although it has been reportedly induced in inflammatory stimuli including lipopolysaccharide, mostly with cultured cells in vitro (30). Intracranial injection of lipopolysaccharide into the substantial nigra is reported to cause dopaminergic neuronal death with a transient increase in COX-2 expression (31), but the cell types expressing COX-2 have not yet been identified. The authors of that study used an approximately 4-fold higher dose of lipopolysaccharide than we used, and so the dosage or the site of injection may contribute to COX-2 induction in neural cells under in vivo conditions. In agreement with our results, COX-2 expression in neuronal cells but not in microglia or astrocytes was also reported in animal models and in patients with neurodegeneration such as that caused by Parkinson disease and Alzheimer disease $(32,11)$.

${ }^{11} \mathrm{C}$-ketoprofen methyl ester showed high accumulation into the inflamed area at the early phase after lipopolysaccharide injection, and the time dependency corresponded well with the population of COX-1-expressing activated microglia and macrophages. The increased number of COX1-expressing microglia and macrophages was approximately $40 \%$ of the total number of COX-1-expressing cells, which is not significant in protein level, though increased ${ }^{11} \mathrm{C}$-ketoprofen methyl ester accumulation was much higher than that of the corresponding radioactivity related to this increase (3.7-fold from the basal level). Thus, not only expression but also elevated enzymatic activity of COX-1 might be related to the changes in radioactivity of ${ }^{11} \mathrm{C}$-ketoprofen methyl ester observed in the PET study.

Recently, it has been reported that pharmacologic inhibition and gene disruption of COX-1 in mice decreases the activation of microglia and astrocytes induced by the intracerebroventricular injection of lipopolysaccharide with reduction of proinflammatory cytokines, chemokines, and oxidative stress (33). However, COX-2-deficient mice show exacerbation of neuronal damage with an increase of proinflammatory agents and oxidative stress in response to lipopolysaccharide (34). In addition, neuroinflammationinduced blood-brain barrier breakdown induced by lipopolysaccharide has also been shown to be attenuated by COX-1 inhibition yet was not prevented by COX-2 inhib- 
ition (35). Supporting our data, these studies indicate that COX-1 plays proinflammatory roles in neuroinflammatory processes. It has also been reported that COX-1-positive microglia are largely present in neuronal diseases, such as Alzheimer disease, Parkinson disease, and traumatic brain injury, indicating the involvement of COX-1 via activation of microglia in the pathologic process of these neurodegenerative disorders $(8-11)$. Therefore, COX-1 might be a critical target for the diagnosis and therapeutic treatment of neurodegenerative disorders.

The elevation of ${ }^{11} \mathrm{C}$-ketoprofen methyl ester accumulation and COX-1-expressing activated microglia and macrophages were observed in neuroinflammation induced by both lipopolysaccharide and quinolinic acid, though the mechanisms of these compounds were quite different. It is well established that lipopolysaccharide is a stimulant of the innate immune system, recognized by TLR4, and induces a robust inflammatory response by activating phagocytic cells such as microglia $(16,17)$. Injection of quinolinic acid induced significant neuronal death in the rat striatum, along with activation of microglia and macrophages expressing COX-1 - a result that is similar to the results obtained for lipopolysaccharide. However, quinolinic acid has activated astrocytes different from lipopolysaccharide in the early phase, and no expression of COX-1 or COX-2 in these astrocytes was observed. Quinolinic acid is reported to induce direct damage of neuronal cells and astrocytes by its excitatory effect, with microglial activation seemingly a secondary reaction of this cell death $(18,19)$. The involvement of COX-2 rather than COX-1 in neuronal damage induced by quinolinic acid has previously been reported (36). Inhibition of COX-2 but not COX-1 was found to provide structural and functional protection against quinolinic acid-induced neurodegeneration. Different from our experimental conditions, an almost 4-fold higher dose (225 nmol) of quinolinic acid was used in that study, and the authors evaluated the effects at $27 \mathrm{~d}$ after the quinolinic acid injection. Therefore, COX-2 might be involved in the later phase of inflammation associated with reactive gliosis, caused by a robust insult, rather than the early phase of initiation of proinflammatory factors.

The time-dependent changes of ${ }^{11} \mathrm{C}$-ketoprofen methyl ester and ${ }^{11} \mathrm{C}-\mathrm{PK} 11195$ were quite different in the rat striatum after the injection of lipopolysaccharide. High accumulation of ${ }^{11} \mathrm{C}$-ketoprofen methyl ester was observed in the early phase, corresponding well to the time course of activation of microglia and macrophages, as demonstrated by immunohistochemistry. Different from our results, the results of some animal model studies of ischemia and also lipopolysaccharide injection with a higher dosage (100fold) showed strong and prolonged activation of microglia after injury $(37,38)$. The time course of activation of microglia and macrophages might depend on the nature or intensity of the injury. In contrast, the high accumulation of ${ }^{11} \mathrm{C}-\mathrm{PK} 11195$ started slowly and lasted until at least $14 \mathrm{~d}$ afterward. Because ${ }^{11} \mathrm{C}-\mathrm{PK} 11195$ is known to be specific to translocator protein, which is highly expressed in activated microglia and astrocytes $(39,40)$, the time course of ${ }^{11} \mathrm{C}$ PK11195 might correspond to changes in both activation of microglia/macrophages and astrocytes. As a preliminary result, we also observed the appearance of translocator protein/OX-42- and translocator protein/GFAP-positive cells at the early and late phases, respectively (Supplemental Fig. 7). Therefore, ${ }^{11} \mathrm{C}$-ketoprofen methyl ester is highly responsible for and sensitive to the neuroinflammatory processes targeting COX-1 in activated microglia and macrophages.

Hydrolysis of ${ }^{11} \mathrm{C}$-ketoprofen methyl ester possibly influences its brain accumulation. However, in rats, ${ }^{11} \mathrm{C}$-ketoprofen methyl ester that incorporates into the brain is hydrolyzed rapidly (15), and levels of ${ }^{11} \mathrm{C}$-ketoprofen in normal and inflamed brain tissues at $2 \mathrm{~min}$ after ${ }^{11} \mathrm{C}$-ketoprofen methyl ester administration have shown no significant difference $(70.3 \% \pm 1.4 \%$ vs. $80.5 \% \pm 6.5 \%, n=3, P>0.05)$ at day 1 after lipopolysaccharide injection. Although further studies on quantitative analysis with kinetic models are required for clinical application, ${ }^{11} \mathrm{C}$-ketoprofen methyl ester could act as a valuable tool for investigating the role of COX1 and for diagnosing the pathologic process of neurodegenerative disorders.

\section{CONCLUSION}

We have identified ${ }^{11} \mathrm{C}$-ketoprofen methyl ester as a COX-1-selective PET probe and have succeeded in visualizing the expression of COX-1 in microglia and macrophages during the neuroinflammatory process in the living brain. Time-dependent in vivo expression of COX-1 in activated microglia and macrophages during neuroinflammation, which was revealed by our PET study of ${ }^{11} \mathrm{C}$-ketoprofen methyl ester in rats, strongly indicates a specific action of COX-1 in the neuroinflammatory process. COX-1 may play a crucial role in the pathology of neuroinflammation and might be a critical target for the diagnosis and therapy of neurodegenerative disorders.

\section{DISCLOSURE STATEMENT}

The costs of publication of this article were defrayed in part by the payment of page charges. Therefore, and solely to indicate this fact, this article is hereby marked "advertisement" in accordance with 18 USC section 1734.

\section{ACKNOWLEDGMENTS}

This work was supported in part by a consignment expense for the Molecular Imaging Research Programs titled "Research Base for Exploring New Drugs" and "Kobe Cluster" from the Ministry of Education, Culture, Sports, Science and Technology of the Japanese government; a Health and Labour Sciences Research Grant for Research on Regenerative Medicine for Clinical Application; and a Grant-in-Aid for Young Scientists (B) of the Japan Society for the Promotion of Science. No other potential conflict of 
interest relevant to this article was reported. We thank Masahiro Kurahashi (Sumitomo Heavy Industry Accelerator Service Ltd.), Yumiko Katayama, Miki Goto, Yasuhiro Wada, and Emi Hayashinaka (RIKEN) for their assistance with the PET study.

\section{REFERENCES}

1. Cartier L, Hartley O, Dubois-Dauphin M, Krause KH. Chemokine receptors in the central nervous system: role in brain inflammation and neurodegenerative diseases. Brain Res Brain Res Rev. 2005;48:16-42.

2. Glass CK, Saijo K, Winner B, Marchetto MC, Gage FH. Mechanisms underlying inflammation in neurodegeneration. Cell. 2010;140:918-934.

3. Phillis JW, Horrocks LA, Farooqui AA. Cyclooxygenases, lipoxygenases, and epoxygenases in CNS: their role and involvement in neurological disorders. Brain Res Rev. 2006;52:201-243.

4. Farooqui AA, Horrocks LA, Farooqui T. Modulation of inflammation in brain: a matter of fat. J Neurochem. 2007;101:577-599.

5. Smith WL, Garavito RM, DeWitt DL. Prostaglandin endoperoxide H synthases (cyclooxygenases)-1 and -2. J Biol Chem. 1996;271:33157-33160.

6. Bazan NG, Fletcher BS, Herschman HR, Mukherjee PK. Platelet-activating factor and retinoic acid synergistically activate the inducible prostaglandin synthase gene. Proc Natl Acad Sci USA. 1994;91:5252-5256.

7. Minghetti L. Cyclooxygenase-2 (COX-2) in inflammatory and degenerative brain diseases. J Neuropathol Exp Neurol. 2004;63:901-910.

8. Choi SH, Aid S, Bosetti F. The distinct roles of cyclooxygenase-1 and -2 in neuroinflammation: implications for translational research. Trends Pharmacol Sci. 2009;30:174-181.

9. Schwab JM, Nguyen TD, Postler E, Meyermann R, Schluesener HJ. Selective accumulation of cyclooxygenase-1-expressing microglial cells/macrophages in lesions of human focal cerebral ischemia. Acta Neuropathol. 2000;99:609-614.

10. Schwab JM, Beschorner R, Meyermann R, Gözalan F, Schluesener HJ. Persistent accumulation of cyclooxygenase-1-expressing microglial cells and macrophages and transient upregulation by endothelium in human brain injury. $J$ Neurosurg. 2002;96:892-899.

11. Hoozemans JJ, Rozemuller JM, van Haastert ES, Veerhuis R, Eikelenboom P. Cyclooxygenase-1 and -2 in the different stages of Alzheimer's disease pathology. Curr Pharm Des. 2008;14:1419-1427.

12. de Vries EF. Imaging of cyclooxygenase-2 (COX-2) expression: potential use in diagnosis and drug evaluation. Curr Pharm Des. 2006;12:3847-3856.

13. de Vries EF, van Waarde A, Buursma AR, Vaalburg W. Synthesis and in vivo evaluation of ${ }^{18} \mathrm{~F}$-desbromo-DuP-697 as a PET tracer for cyclooxygenase-2 expression. J Nucl Med. 2003;44:1700-1706.

14. Gao M, Wang M, Miller KD, Hutchins GD, Zheng QH. Synthesis of carbon-11 labeled celecoxib derivatives as new candidate PET radioligands for imaging of inflammation. Appl Radiat Isot. 2009;67:2019-2024.

15. Takashima-Hirano M, Shukuri M, Takashima T, et al. General method for the ${ }^{11} \mathrm{C}$-labeling of 2-arylpropionic acids and their esters: construction of a PET tracer library for a study of biological events involved in COXs expression. Chemistry. 2010;16:4250-4258.

16. Kim WG, Mohney RP, Wilson B, Jeohn GH, Liu B, Hong JS. Regional difference in susceptibility to lipopolysaccharide-induced neurotoxicity in the rat brain: role of microglia. J Neurosci. 2000;20:6309-6316.

17. Rivest S. Molecular insights on the cerebral innate immune system. Brain Behav Immun. 2003;17:13-19.

18. Duan WM, Widner H, Cameron RM, Brundin P. Quinolinic acid-induced inflammation in the striatum does not impair the survival of neural allografts in the rat. Eur J Neurosci. 1998;10:2595-2606.

19. Schwarcz R, Pellicciari R. Manipulation of brain kynurenines: glial targets, neuronal effects, and clinical opportunities. J Pharmacol Exp Ther. 2002;303: $1-10$.

20. Shah F, Hume SP, Pike VW, Ashworth S, McDermott J. Synthesis of the enantiomers of [N-methyl- $\left.{ }^{11} \mathrm{C}\right] \mathrm{PK} 11195$ and comparison of their behaviours as radioligands for PK binding sites in rats. Nucl Med Biol. 1994;21:573581.

21. Guide for the Care and Use of Laboratory Animals. Washington, DC: Government Printing Office; 1985. NIH publication 86-23.

22. Langenbach R, Morham SG, Tiano HF, et al. Prostaglandin synthase 1 gene disruption in mice reduces arachidonic acid-induced inflammation and indomethacin-induced gastric ulceration. Cell. 1995;83:483-492.

23. Morham SG, Langenbach R, Loftin CD, et al. Prostaglandin synthase 2 gene disruption causes severe renal pathology in the mouse. Cell. 1995;83: 473-482.

24. Paxinos G, Watson C. The Rat Brain in Stereotaxic Coordinates. 6th ed. Burlington, MA: Academic Press; 2007.

25. Warner TD, Giuliano F, Vojnovic I, Bukasa A, Mitchell JA, Vane JR. Nonsteroid drug selectivities for cyclo-oxygenase-1 rather than cyclo-oxygenase-2 are associated with human gastrointestinal toxicity: a full in vitro analysis. Proc Natl Acad Sci USA. 1999;96:7563-7568.

26. Chandrasekharan NV, Dai H, Roos KL, et al. COX-3, a cyclooxygenase-1 variant inhibited by acetaminophen and other analgesic/antipyretic drugs: cloning, structure, and expression. Proc Natl Acad Sci USA. 2002;99:13926-13931.

27. Thomas DM, Kuhn DM. Cyclooxygenase-2 is an obligatory factor in methamphetamine-induced neurotoxicity. J Pharmacol Exp Ther. 2005;313:870-876.

28. Shankaran M, Marino ME, Busch R, et al. Measurement of brain microglial proliferation rates in vivo in response to neuroinflammatory stimuli: application to drug discovery. J Neurosci Res. 2007;85:2374-2384.

29. Zito MA, Koennecke LA, McAuliffe MJ, McNally B, van Rooijen N, Heyes MP. Depletion of systemic macrophages by liposome-encapsulated clodronate attenuates striatal macrophage invasion and neurodegeneration following local endotoxin infusion in gerbils. Brain Res. 2001;892:13-26.

30. Bauer MK, Lieb K, Schulze-Osthoff K, et al. Expression and regulation of cyclooxygenase-2 in rat microglia. Eur J Biochem. 1997;243:726-731.

31. de Meira Santos Lima M, Braga Reksidler A, Marques Zanata S, Bueno Machado H, Tufik S, Vital MA. Different parkinsonism models produce a time-dependent induction of COX-2 in the substantia nigra of rats. Brain Res. 2006;1101:117-125.

32. Teismann P, Tieu K, Choi DK, et al. Cyclooxygenase-2 is instrumental in Parkinson's disease neurodegeneration. Proc Natl Acad Sci USA. 2003;100:54735478.

33. Choi SH, Langenbach R, Bosetti F. Genetic deletion or pharmacological inhibition of cyclooxygenase-1 attenuate lipopolysaccharide-induced inflammatory response and brain injury. FASEB J. 2008;22:1491-1501.

34. Aid S, Langenbach R, Bosetti F. Neuroinflammatory response to lipopolysaccharide is exacerbated in mice genetically deficient in cyclooxygenase-2. J Neuroinflammation. 2008;5:17-30.

35. Aid S, Silva AC, Candelario-Jalil E, Choi SH, Rosenberg GA, Bosetti F. Cyclooxygenase-1 and -2 differentially modulate lipopolysaccharide-induced bloodbrain barrier disruption through matrix metalloproteinase activity. J Cereb Blood Flow Metab. 2010;30:370-380.

36. Salzberg-Brenhouse HC, Chen EY, Emerich DF, et al. Inhibitors of cyclooxygenase-2, but not cyclooxygenase-1 provide structural and functional protection against quinolinic acid-induced neurodegeneration. J Pharmacol Exp Ther. 2003; 306:218-228.

37. Rojas S, Martín A, Arranz MJ, et al. Imaging brain inflammation with $\left[{ }^{11} \mathrm{C}\right]$ PK11195 by PET and induction of the peripheral-type benzodiazepine receptor after transient focal ischemia in rats. J Cereb Blood Flow Metab. 2007;27:19751986.

38. Venneti S, Lopresti BJ, Wang G, et al. A comparison of the high-affinity peripheral benzodiazepine receptor ligands DAA1106 and (R)-PK11195 in rat models of neuroinflammation: implications for PET imaging of microglial activation. J Neurochem. 2007;102:2118-2131.

39. Venneti S, Lopresti BJ, Wiley CA. The peripheral benzodiazepine receptor (translocator protein $18 \mathrm{kDa}$ ) in microglia: from pathology to imaging. Prog Neurobiol. 2006;80:308-322.

40. Chen MK, Baidoo K, Verina T, Guilarte TR. Peripheral benzodiazepine receptor imaging in CNS demyelination: functional implications of anatomical and cellular localization. Brain. 2004;127:1379-1392. 